\title{
GPU IMPLEMENTATION OF A VISCOUS FLOW SOLVER ON UNSTRUCTURED GRIDS
}

\author{
XU TIANHAO \\ College of Aerospace Engineering, Nanjing University of Aeronautics and Astronautics, 29 Yudao St., Nanjing \\ Nanjing, 210016, China \\ xthnuaa@163.com \\ CHEN LONG \\ College of Aerospace Engineering, Nanjing University of Aeronautics and Astronautics, 29 Yudao St., Nanjing \\ Nanjing, 210016, China \\ lchen@nuaa.edu.cn
}

Published 22 June 2016

\begin{abstract}
Graphics processing units have gained popularities in scientific computing over past several years due to their outstanding parallel computing capability. Computational fluid dynamics applications involve large amounts of calculations, therefore a latest GPU card is preferable of which the peak computing performance and memory bandwidth are much better than a contemporary high-end CPU. We herein focus on the detailed implementation of our GPU targeting Reynolds-averaged Navier-Stokes equations solver based on finite-volume method. The solver employs a vertexcentered scheme on unstructured grids for the sake of being capable of handling complex topologies. Multiple optimizations are carried out to improve the memory accessing performance and kernel utilization. Both steady and unsteady flow simulation cases are carried out using explicit Runge-Kutta scheme. The solver with GPU acceleration in this paper is demonstrated to have competitive advantages over the CPU targeting one.
\end{abstract}

Keywords: GPU acceleration; Reynolds-averaged Navier-Stokes equations; unstructured grids; vertex-centered scheme.

\section{Introduction}

Over the last few years, it is becoming increasingly common to use a graphics processing unit (GPU) as a modified form of stream processor in scientific parallel computing. Compute Unified Device Architecture (CUDA) is a GPU-based parallel programming model created by NVIDIA in 2007. CUDA makes it easy for scientists and engineers to fully exploit GPU's high performance in parallel computing.

In the field of computational fluid dynamics (CFD), some scholars started to use GPU to accelerate their applications based on Euler equations or Navier-Stokes equations and achieved good results ${ }^{1-3}$. Further implementations on Reynolds-averaged Navier-Stokes

This is an Open Access article published by World Scientific Publishing Company. It is distributed under the terms of the Creative Commons Attribution 3.0 (CC-BY) License. Further distribution of this work is permitted, provided the original work is properly cited. 
(RANS) equations, Large Eddy Simulation (LES) and Direct Numerical Simulation (DNS) were set forth ${ }^{4-6}$. In order to carry out large-scale numerical simulation on GPU, implementations on multiple GPUs have been presented in ${ }^{8,24-25}$. It's then important to improve single GPU performance before realizing multi-GPU version. In the papers mentioned above, structured grids are mostly employed due to their fixed geometry pattern, which makes it easy for researchers to achieve better performance on GPU. On unstructured grids, especially when a vertex-centered scheme is employed, more efforts are needed to improve the kernel performance.

In this paper, a single GPU implementation of the RANS equations solver for unstructured grids based on finite volume method will be presented. The vertex-centered scheme is employed here. To improve the performance, multiple optimization strategies will be discussed. The rest of this paper will be organized as follows. Section 2 introduces the numerical methods used in this paper. Section 3 discusses the detailed GPU implementation and multiple optimization strategies. Section 4 presents several numerical results. Section 5 gives evaluations of this GPU implementation. Section 6 provides some final conclusions and directions for future work.

\section{Numerical Methods}

\subsection{Governing equations}

In a Cartesian coordinate system, the RANS equations can be expressed as

$$
\frac{\partial}{\partial t} \int_{\Omega} \boldsymbol{W} \mathrm{d} \Omega+\oint_{\partial \Omega}\left(\boldsymbol{F}_{c}-\boldsymbol{F}_{v}\right) \mathrm{d} S=0,
$$

where $\boldsymbol{W}$ is the vector of conservative variables, $\boldsymbol{F}_{c}$ is the vector of convective fluxes and $\boldsymbol{F}_{v}$ is the vector of the viscous fluxes.

These equations are discretized in space on an unstructured grid using a vertexcentered finite volume method as shown in Fig. 1. The discretization of the governing equations Eq. (1) separately for spatial and temporal, written down for each control volume, forms a set of equations in time

$$
\Omega_{I} \frac{\mathrm{d} \boldsymbol{W}_{I}}{\mathrm{~d} t}=-\boldsymbol{R}_{I}=-\sum_{m=1}^{N_{F}}\left(\boldsymbol{F}_{c}-\boldsymbol{F}_{v}\right)_{m} \Delta S_{m},
$$

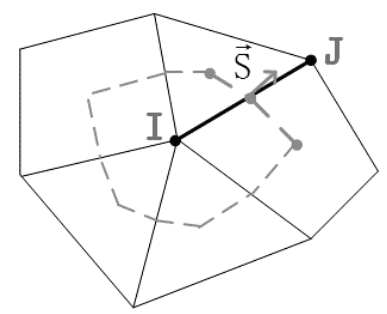

Fig. 1. Vertex-centered scheme for unstructured grids. 


\subsection{Numerical schemes}

A Roe's approximate Riemann solver ${ }^{14}$ is used to compute the convective fluxes

$$
\boldsymbol{F}_{c}=\frac{1}{2}\left[\boldsymbol{F}_{c}\left(\boldsymbol{W}_{L}\right)+\boldsymbol{F}_{c}\left(\boldsymbol{W}_{R}\right)-\left|A_{R o e}\right|\left(\boldsymbol{W}_{R}-\boldsymbol{W}_{L}\right)\right] \text {. }
$$

To achieve second order spatial accuracy on regular grids, a piecewise linear reconstruction is employed to evaluate the left and right states

$$
\boldsymbol{W}_{L}=\boldsymbol{W}_{I}+\frac{1}{2} \Psi_{I}\left(\nabla \boldsymbol{W}_{L} \cdot \boldsymbol{r}_{I J}\right) \text { and } \boldsymbol{W}_{R}=\boldsymbol{W}_{J}-\frac{1}{2} \Psi_{J}\left(\nabla \boldsymbol{W}_{R} \cdot \boldsymbol{r}_{I J}\right) \text {, }
$$

where $\Psi$ is Venkatakrishnan's limiter used to prevent the generation of oscillations and spurious solutions in regions with large gradients (e.g., at shocks).

A Green-Gauss theorem based approach is applied to evaluate the gradients at a node

$$
\nabla U \approx \frac{1}{\Omega} \int_{\partial \Omega} U \boldsymbol{n} \mathrm{d} S=\frac{1}{\Omega} \sum_{j=1}^{N_{F}} \frac{1}{2}\left(U_{i}+U_{j}\right) \boldsymbol{n}_{i j} \Delta S_{i j} .
$$

Time marching for steady flows is performed using an explicit multi-stage scheme first presented by Jameson et al. ${ }^{15}$.

To accelerate the convergence, a local time-stepping technique ${ }^{20}$ is employed.

Dual time-stepping approach ${ }^{18}$ is implemented using the explicit multistage scheme for unsteady flows, which is second-order time accurate.

The Spalart-Allmaras one-equation model ${ }^{16}$ is introduced here as a first-order closure. The integral form can be organized as

$$
\frac{\partial}{\partial t} \int_{\Omega} \tilde{v} \mathrm{~d} \Omega+\oint_{\partial \Omega}\left(F_{c, T}-F_{v, T}\right) \mathrm{d} S=\int_{\Omega} Q_{T} \mathrm{~d} \Omega .
$$

Then we can solve the turbulence model equation Eq. (6) with a similar scheme applied to the governing equations Eq. (1).

\section{GPU Implementation}

\section{1. $G P U$ architecture}

A GPU consists of an array of Streaming Multiprocessors (SM), each of which is capable of supporting thousands of co-resident concurrent hardware threads. In CUDA, 32 threads are grouped into a warp while a couple of warps are grouped into a block. A warp executing one common instruction and a block sharing same limited on-chip resources at a time are two primary features of GPU programming model.

The GPU used in this paper is NVIDIA Tesla K40 which has 12GB device memory, allowing large-scale numerical simulation. Tesla K40's compute capability is 3.5 and devices of compute capability below 3.5 are not considered here, which means implementation in this paper may not work on older devices. 


\subsection{Data layout}

In CPU implementation, the Array of Structures (AoS) data layout is generally employed to achieve high memory access performance. However a Structure of Arrays (SoA) data layout is more suitable for GPU Implementation due to the data-parallel feature of GPU. Contiguous threads within a warp will execute a memory access instruction simultaneously, which allows coalescing memory access if SoA data layout is employed as shown in Fig. 2. As discussed in introduction, implementation on structured grids allows organized memory access, thus, higher speed-ups. When the spatial discretization is carried out on unstructured grids, the techniques used to achieve higher memory access performance and to avoid memory conflicts differ. If a cell-centered scheme is used, a fixed number of neighboring cells depending on cell type allows an implementation with no memory conflict and better memory access performance ${ }^{4}, 7$. However, a vertexcentered scheme is used in this paper, thus, arousing additional efforts to deal with the memory.

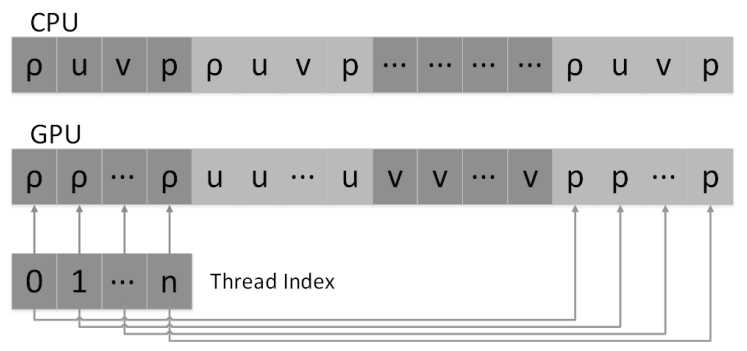

Fig. 2. Data layouts on CPU and GPU.

\subsection{Kernel design}

Since all calculating related data are transferred from host (CPU) to device (GPU) once at the beginning and will be partly transferred back only if the iterative procedure is terminated, no PCI-E related optimization issue will be discussed here.

Implementation flow chart of the solver for CPU and GPU is shown in Fig. 3. The additional steps for GPU with dashed lines are to transfer data between CPU and GPU before and after the main loop on GPU. In this case, a direct kernel design is described in Fig. 4, where kernels are mapped to the processes within the main loop of the flow chart. In Fig. 4, node-based means that a thread is associated with a node while edge-based means a thread is associated with an edge. However, this implementation will apparently lead to memory conflicts in edge-based kernels when several threads try to write to the same address (for instance, in the kernel for computing fluxes, several edge-associated threads will possibly add fluxes to a same node simultaneously). A method is to use atomic operations. It should be noted that atomicAdd() in CUDA only supports data of int or float type. An alternative way to overload atomicAdd() for data of double type is to use atomicCAS(). Then this method can be realized with $\mathrm{CUDA} \mathrm{C} / \mathrm{C}++$. Note that a 
multi-color method is evaluated in Ref. 20 which proves to be not efficient at all when a vertex-centered discretization scheme is applied.

With no global synchronization instruction in CUDA, the split of kernels plays the role as a global barrier. Since a Roe's approximate Riemann solver is employed here, relatively high arithmetic intensity will compensate for the additional global memory accesses caused by separate kernels.

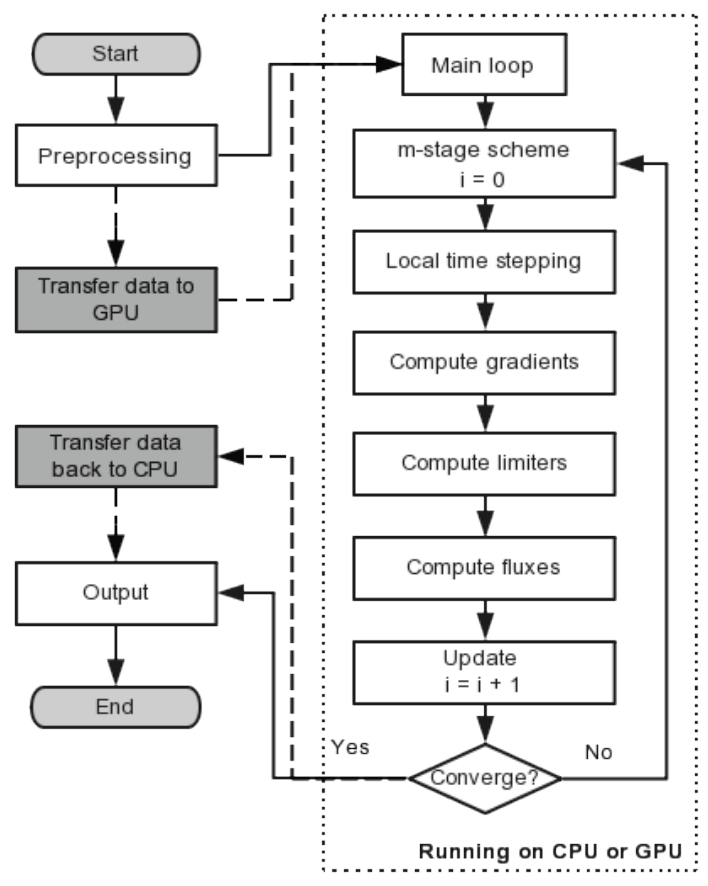

Fig. 3. Flow chart of the solver for CPU and GPU.

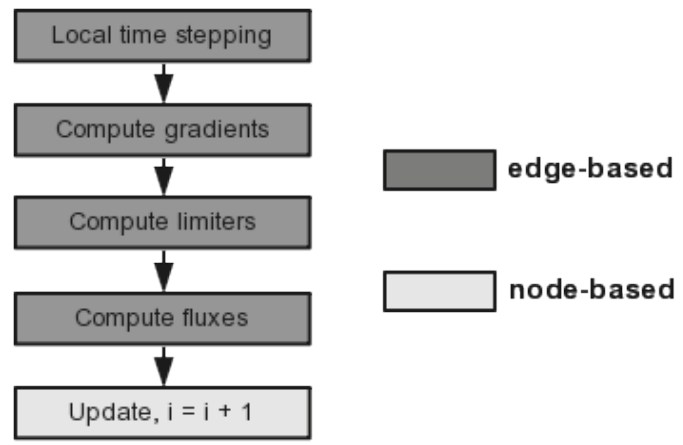

Fig. 4. Design of the kernels on GPU. 


\subsection{Optimizations}

\subsubsection{Optimize memory usage}

In such an application that involves many global memory transactions, maximizing coalescing leads to maximized memory throughput. In edge-based kernels in Fig. 4, low global memory throughput will be achieved when fetching data associated with two end nodes of an edge which are not successive in numbering. This problem makes it difficult to fulfill a vertex-centered scheme on GPU efficiently. With the development of NVIDIA GPU hardware, a larger L2 cache alleviates the demand for coalescing. Moreover, data in double precision is more easily to be aligned to 32-, 64- or 128-byte segments of device memory. Therefore maximizing memory throughput is equivalent to making nodes close to each other in data locality. In traditional CPU codes, multiple grid reordering methods are developed to minimize the frequency of cache-misses. One of the methods is reverse Cuthill-McKee (RCM) reordering ${ }^{22}$, which is generally used to reduce matrix bandwidth. It also performs well on GPU for vertex-centered scheme. In addition, we present another approach to improve data locality by using METIS $^{23}$ partitioning package. In this approach, grids are divided into small partitions containing 128 (or other small numbers) nodes. Then all the nodes and edges are renumbered by the sequence of these partitions. The second approach achieves better performance than RCM reordering in most cases. However, when the grids are in large scale, the hardware requirements and execution time can be unacceptable, thus, the RCM reordering is preferred in this case.

\subsubsection{Maximize utilization}

The number of blocks and warps that can reside and be processed together on the multiprocessor for a given kernel depends on the amount of registers and shared memory used by the kernel. Shared memory will not be used in this paper and thus the register usage is the only bottleneck of the warp occupancy. With the help of Occupancy Calculator released by NVIDIA, it's easy to calculate warp occupancy by the given register and shared memory usage. Higher warp occupancy can be achieved if less registers are used ${ }^{21}$. We can restrict the register usage by passing -maxrregcount flag to nvcc compiler or using _ launch_bounds_ qualifier in kernel declaration. Restricting register usage will cause register spills, which will be partly cached in L1 and partly stored in global memory. A $20 \%$ improvement is achieved by properly restricting the registers in this paper.

Another method to maximize the utilization is to find out the best block size. It can be easily carried out by testing several block sizes listed in Occupancy Calculator which ensure highest occupancy. In this paper, 64 and 128 are chosen as block sizes of different kernels.

\section{Numerical Results}

Three cases are presented in this section to test our solver's GPU implementation. 


\subsection{RAE2822 transonic airfoil}

This case demonstrates the solver's ability to simulate turbulent, transonic flow over the RAE 2822 airfoil, corresponding to Case 6 from Ref. 26. A wind tunnel experiment result is given by Ref. 26. Numerical results from the documented validation case for the NPARC code is also used as a comparison.

Free stream conditions of this case are summarized in Table 1.

Table 1 Free stream conditions of RAE2822 transonic airfoil case.

\begin{tabular}{lll}
\hline Mach & Reynolds number & Angle of Attack $(\mathrm{deg})$ \\
\hline 0.729 & $6,500,000$ & 2.31 \\
\hline
\end{tabular}

A 3D grid is simply extruded from the 2D grid because our solver is for $3 \mathrm{D}$ flows. Pressure coefficient of the lower and upper surfaces is plotted in Fig. 5. The numerical result agrees well with experimental measurements and NPARC's numerical result.

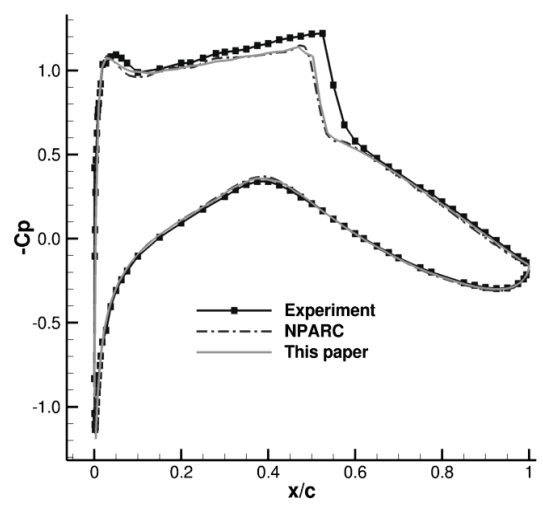

Fig. 5. Pressure coefficient of the lower and upper surfaces.

\subsection{ONERA M6 wing}

Flow over ONERA M6 wing is simulated in this paper as a validation case for 3D external flows. It is a classic CFD test case due to its simple geometry combined with complexities of transonic flow, involving local supersonic flow, shocks and turbulent boundary layers separation. Experiment results are documented in Ref. 27, which will be used here for comparison. The flow is at a free-stream Mach number of 0.8395, an angle of attack of 3.06 degrees, an angle of sideslip of 0 degree, and a Reynolds number of 11.72 million in this case. The pressure contours on the wing surface are shown in Fig. 6, combing with the surface grid. Experiment results are given as pressure coefficients at seven span-wise sections, of which the locations are specified with respect to the wing span $b$, listed in Table 2 . 
Table 2 Span-wise locations of the sections.

\begin{tabular}{cccccccc}
\hline Section & 1 & 2 & 3 & 4 & 5 & 6 & 7 \\
\hline $\mathrm{y} / \mathrm{b}$ & 0.2 & 0.44 & 0.65 & 0.8 & 0.9 & 0.95 & 0.99 \\
\hline
\end{tabular}

Then the numerical results consist of pressure coefficients of these sections are shown in Fig. 7 along with the experiment results.

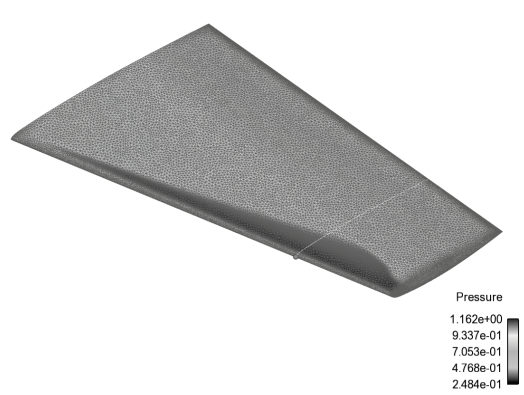

Fig. 6. Pressure contours on the wing surface, combing with the surface grid.

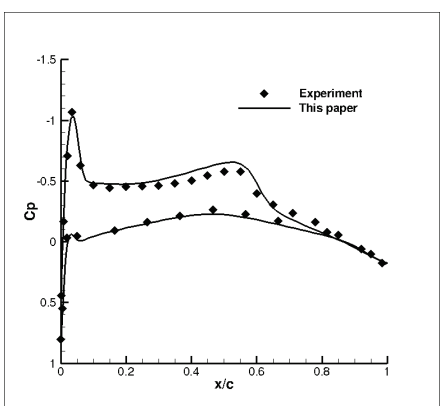

(a) Section 1

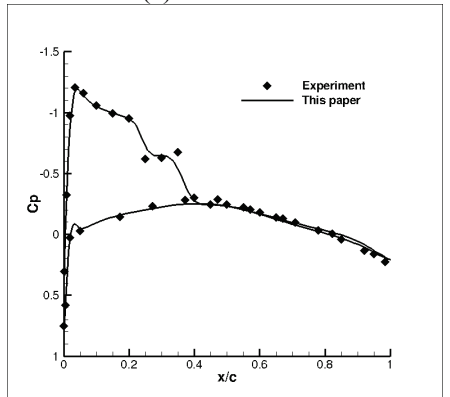

(d) Section 4

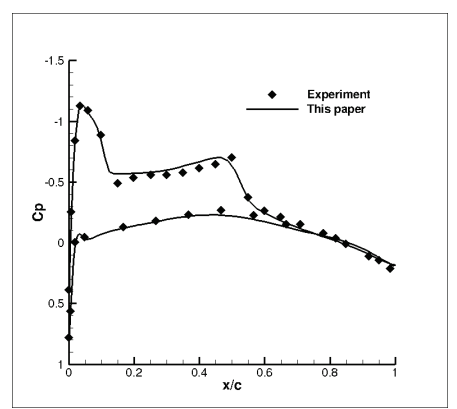

(b) Section 2

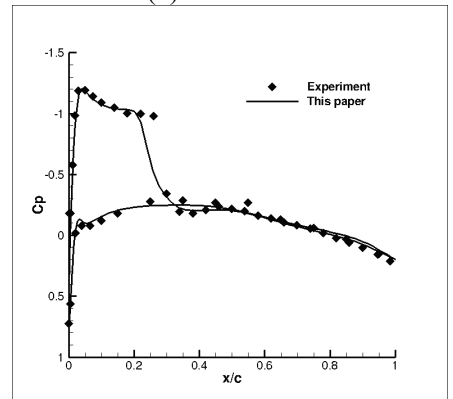

(e) Section 5

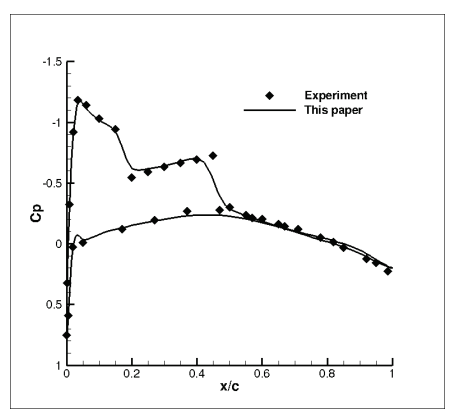

(c) Section 3

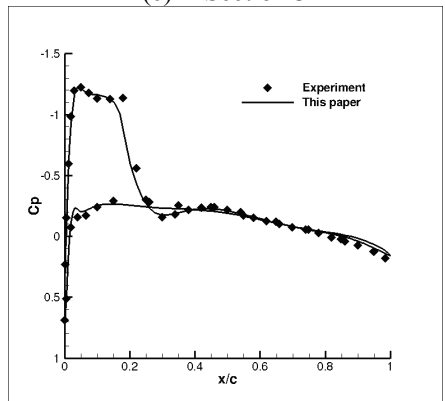

(f) Section 6 


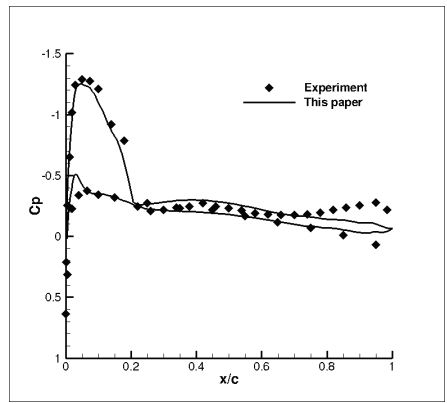

(g) Section 7

Fig. 7. Pressure coefficients of the selected sections.

Reasonable agreement with the experiment data is obtained at these sections while minor difference of the shock wave location occur at section 4-6, showing the difficulties in precisely predicting transonic flow.

\subsection{Von Kármán Vortex Shedding}

Von Kármán Vortex Shedding indicates the phenomena that pairs of alternate vortices periodically detach from a bluff-body, generating a vortex street. In this case, the laminar flow will pass a perpendicular cylinder, of which the conditions are defined in Table 3 such that the vortex shedding will take place in the numerical simulation.

Table 3 Free stream conditions of Von Kármán Vortex Shedding case.

\begin{tabular}{lll}
\hline Mach & Reynolds number & Angle of Attack $($ deg $)$ \\
\hline 0.2 & 200 & 0 \\
\hline
\end{tabular}

Grid used in this case is generated in a way similar to that in the first case and the cylinder diameter is set to one. Fig. 8 shows the instantaneous velocity contours of the flow field, in which the pairs of alternate vortices are predicted. A Strouhal number of 0.196 is obtained with respect to the cylinder diameter. A reasonable result has been achieved, which demonstrates the solver's capability of simulating unsteady flows.

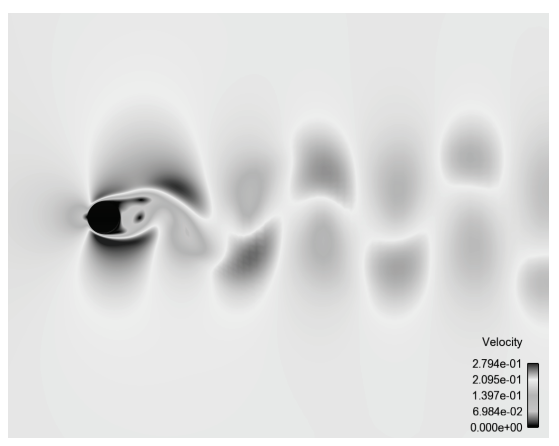

Fig. 8. Instantaneous velocity contours of the flow field. 


\section{Performance Evaluations}

Performance evaluations are briefly discussed in this section to show the outstanding power of GPU computing. Tesla K40 provides 1.43 TFlop of double precision throughput and $288 \mathrm{~GB} / \mathrm{s}$ memory bandwidth (with ECC off). Intel Xeon Processor E3-1230 v3 is used in this paper, providing 52.8 GFlop of double precision throughput every physical core and $25.6 \mathrm{~GB} / \mathrm{s}$ memory bandwidth.

Since the scale of a grid is one of the factors that determine the code's performance, four set of grids, of which the details are listed below in Table 4, will be used in the evaluation.

Table 4 Scale of the grids used in the evaluations.

\begin{tabular}{lcccc}
\hline Grid & 1 & 2 & 3 & 4 \\
\hline Number of points & 103,192 & 491,038 & $2,031,392$ & $5,019,453$ \\
\hline
\end{tabular}

Before evaluating overall performance, effects of optimizing strategies discussed above will be analyzed. The comparison of RCM reordering and METIS based reordering is shown in Fig. 9(a), where the speedup is relative to calculations without using any reordering method. It's clear that these two methods both greatly improve the solver's performance while the METIS based one is superior to the other. A second evaluation is done to testify the utilization optimization method as shown in Fig. 9(b), where an average speed-up of 1.2 has been achieved. The overall performance is evaluated by employing METIS based reordering method and other optimization strategies presented in this paper. Unstructured grids and structured grids with equivalent number of points are both tested and the results are shown in Fig. 9(c). It is shown that a speedup of 40-60 times for unstructured grids and 70-100 times for structured grids has been obtained respectively, highlighting the promising feature of GPU implementation. Better performance is achieved on structured grids because of more coalescing memory accesses and less memory conflicts caused by accumulating fluxes.

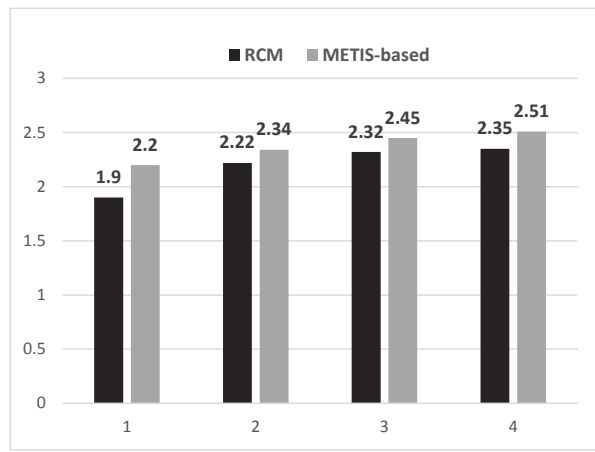

(a) Grid reordering

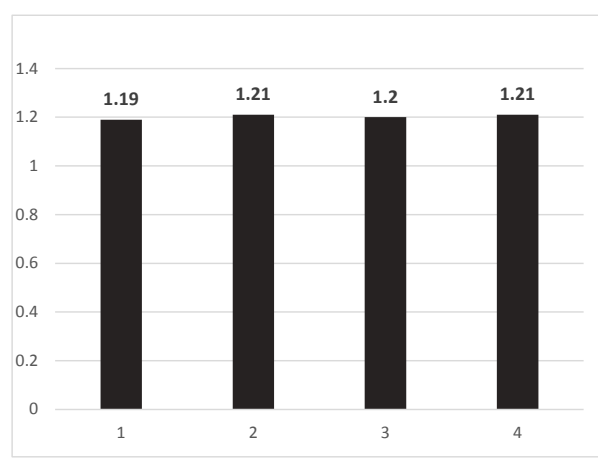

(b) Register optimization 


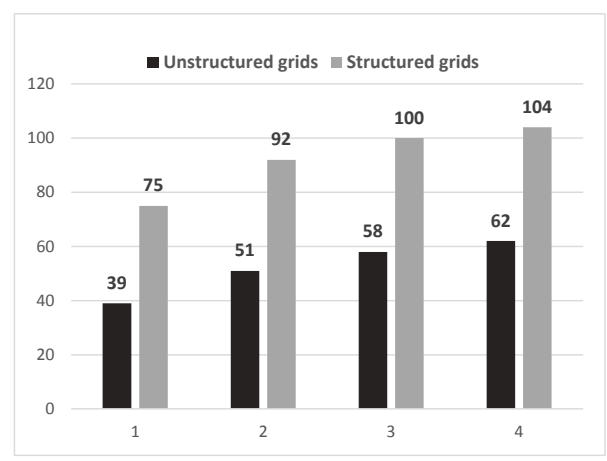

(c) Overall speedup

Fig. 9. Performance evaluations.

\section{Conclusions}

GPU implementation of a viscous flow solver based on unstructured grids is presented in this paper. The detailed realization of a vertex-centered scheme and the strategies to optimize memory usage and device utilizations are discussed. Calculations on a single GPU are around 40-60 times faster than those on a single CPU core for unstructured grids in our work. A CUDA-MPI hybrid multi-GPU implementation is in progress.

\section{Acknowledgments}

We gratefully acknowledge the support of NVIDIA Corporation with the donation of the Tesla K40 GPU used for this research.

\section{References}

1. Hagen, Trond Runar, Knut-Andreas Lie, and Jostein R. Natvig. "Solving the Euler equations on graphics processing units." Computational Science-ICCS 2006. Springer Berlin Heidelberg, 2006. 220-227.OPAL Collab. (G. Abbiendi et al.), Eur. J. Phys. C 11, 217 (1999).

2. Brandvik, Tobias, and Graham Pullan. "Acceleration of a 3D Euler solver using commodity graphics hardware." 46th AIAA aerospace sciences meeting and exhibit. Vol. 607. 2008.

3. Cohen, J., and M. Jeroen Molemaker. "A fast double precision CFD code using CUDA." Parallel Computational Fluid Dynamics: Recent Advances and Future Directions (2009): 414429.

4. Dawes, W. N., P. C. Dhanasekaran, and W. P. Kellar. "Acceleration of an unstructured hybrid mesh RANS solver by porting to GPU architectures." 49th AIAA Aerospace Sciences Meeting Including the New Horizons Forum and Aerospace Exposition. 2011.

5. DeLeon, Rey, Dana Jacobsen, and Inanc Senocak. "Large-eddy simulations of turbulent incompressible flows on GPU clusters." Computing in Science \& Engineering 15.1 (2013): 2633.

6. Khajeh-Saeed, Ali, and J. Blair Perot. "Direct numerical simulation of turbulence using GPU accelerated supercomputers." Journal of Computational Physics 235 (2013): 241-257.

7. Corrigan, Andrew, et al. "Running unstructured grid based CFD solvers on modern graphics hardware." AIAA paper 4001 (2009): 22-25. 
8. Jacobsen, Dana A., Julien C. Thibault, and Inanc Senocak. "An MPI-CUDA implementation for massively parallel incompressible flow computations on multi-GPU clusters." 48th AIAA Aerospace Sciences Meeting and Exhibit. Vol. 16. 2010.

9. Asouti, V. G., et al. "Unsteady CFD computations using vertex - centered finite volumes for unstructured grids on Graphics Processing Units." International Journal for Numerical Methods in Fluids 67.2 (2011): 232-246.

10. Ma Wenpeng, Zhonghua Lu, and Jian Zhang. "GPU parallelization of unstructured/hybrid grid ALE multigrid unsteady solver for moving body problems." Computers \& Fluids (2014).

11. López-Morales, Manuel R., et al. "Verification and Validation of HiFiLES: a High-Order LES unstructured solver on multi-GPU platforms." (2014).

12. Antoniou, Athanasios S., et al. "Acceleration of a finite-difference WENO scheme for largescale simulations on many-core architectures." 48th AIAA Aerospace Sciences Meeting Including the New Horizons Forum and Aerospace Exposition, Orlando, FL, Jan. 2010.

13. Karantasis, Konstantinos I., Eleftherios D. Polychronopoulos, and John A. Ekaterinaris. "High order accurate simulation of compressible flows on GPU clusters over Software Distributed Shared Memory." Computers \& Fluids 93 (2014): 18-29.

14. Roe, Philip L. "Approximate Riemann solvers, parameter vectors, and difference schemes." Journal of computational physics 43.2 (1981): 357-372.

15. Jameson, Antony. "Numerical solution of the Euler equations for compressible inviscid fluids." Numerical methods for the Euler equations of fluid dynamics 1 (1985).

16. Spalart, PHILLIPE R., and Steven R. Allmaras. "A one-equation turbulence model for aerodynamic flows." (1992).

17. Spalart, P. R., et al. "Comments on the feasibility of LES for wings, and on a hybrid RANS/LES approach." Advances in DNS/LES 1 (1997): 4-8.

18. Jameson, Antony. "Time dependent calculations using multigrid, with applications to unsteady flows past airfoils and wings." AIAA paper 1596 (1991): 1991.

19. Osher, Stanley, and Richard Sanders. "Numerical approximations to nonlinear conservation laws with locally varying time and space grids." Mathematics of computation 41.164 (1983): 321-336.

20. Trompoukis, X., et al. "CUDA implementation of vertex-centered, finite volume CFD methods on unstructured grids with flow control applications." GPU Computing Gems 2 (2011): 207224.

21. Cuda, C. "Programming guide." NVIDIA Corporation, July (2012).

22. George, Alan, et al. "Solution of sparse positive definite systems on a hypercube." Journal of Computational and Applied Mathematics 27.1 (1989): 129-156.

23. Karypis, George, and Vipin Kumar. "A fast and high quality multilevel scheme for partitioning irregular graphs." SIAM Journal on scientific Computing 20.1 (1998): 359-392.

24. Castonguay, Patrice, et al. "On the development of a high-order, multi-GPU enabled, compressible viscous flow solver for mixed unstructured grids." AIAA paper 3229 (2011): 2011.

25. DeLeon, Rey, Dana Jacobsen, and Inanc Senocak. "Large-eddy simulations of turbulent incompressible flows on GPU clusters." Computing in Science \& Engineering 15.1 (2013): 2633.

26. Cook, P. H., M. C. P. Firmin, and M. A. McDonald. Aerofoil RAE 2822: pressure distributions, and boundary layer and wake measurements. RAE, 1977.

27. Schmitt, V., and F. Charpin. "Pressure distributions on the ONERA-M6-Wing at transonic Mach numbers." Experimental data base for computer program assessment 4 (1979). 